patients were found to have solitary kidneys-two situated normally and three in various ectopic sites. The appearances of the intravenous pyelograms in three of these cases are shown in Figs. 1 to 4 .

Skeletal abnormalities were found in four patients, three of whom had solitary kidneys. $X$-ray films of two of these cases are shown in Figs. 5 and 6 . Cases 5 and 12 had webbing of the neck on clinical examination. Case 5 had hemivertebrae and spina bifida of the cervical spine and lumbarization of the first sacral segment. Case 12 showed scoliosis of the cervicodorsal spine with a butterfly vertebra at C.7 and congenital fusion of the first and second dorsal vertebrae. Case 13 showed congenital fusion of the left first to eighth ribs and a small scapula. The head of the left humerus, the left radius, and the left radial sector of the hand were absent.

\section{Discussion}

The various skeletal abnormalities associated with absence of the vagina have been given insufficient attention. Of the 11 cases reported from the Johns Hopkins Hospital (Novak and Jones, 1961) only one case was reported as showing a skeletal abnormality, lumbarization of the sacral segment. Bryan et al. (1949) reported six cases showing various skeletal abnormalities among the 100 cases of absence of vagina. These anomalies of the skeleton included congenital dislocation of the hip ; malformations of the foot, arms, and ribs ; cervical spina bifida ; hemivertebrae in the lumbar spine; rudimentary first rib; and sacralization of the fifth lumbar vertebra. A much higher incidence of abnormalities of the skeleton was reported by Chawla et al. (1963). In their series of 18 cases of congenital lesions of the genital tract four showed skeletal abnormality. In the present series four cases showed skeletal anomalies.
Since the incidence of malformations of the urinary tract associated with absence of the vagina is very high, a proper functional and anatomical evaluation of the urinary tract is mandatory before surgery is undertaken. The presence of an ectopic pelvic kidney may interfere with reconstructive surgery. A pelvic kidney and its ureter may be injured during the surgical procedure if its position is not known pre-operatively, as had been emphasized by Pommerenke and Benjamin (1947) and by Woolf and Allen (1953).

\section{Summary}

Twenty-three cases of congenital absence of the vagina are presented. Eleven of these presented various abnormalities of the upper urinary tract, 10 of which were significant. The importance of urinary tract studies is stressed.

Skeletal abnormalities were found in four patients, and it is suggested that insufficient attention has been paid to the incidence of bone abnormality in this condition.

We are grateful to the Editor of the Indian Fournal of Radiology for permission to reproduce Fig. 1.

\section{REFERENCES}

Bryan, A. L., Nigro, J. A., and Counseller, V. S. (1949). Surg. Gynec. Obstet., 88, 79.

Chawla, S., Gadekar, N. G., Nirula, R., and Hingorani, V. (1963). Indian 7. Surg., 25, 600 .

Engstad, J. E. (1917). Lancet, 37, 329.

Novak, E. R., and Jones, G. S. (1961). Textbook of Gynaecology, 6th ed., p. 124. Williams and Wilkins, Baltimore.

Owens, N. (1942). Surgery, 12, 139.

Phelan, J. T., Counseller, V. S., and Green, L. F. (1953). Surg. Gynec. Obstet., 97,1 .

Pommerenke, W. T., and Benjamin, J. A. (1947). N.Y. St. F. Med., 47,

Woolf, R. B., and Allen, W. M. (1953). Obstet. and Gynec., 2, 236.

\title{
Bronchopulmonary Geotrichosis with Severe Asthma
}

\author{
J. D. ROSS,* M.B., CH.B., F.R.C.P.ED., M.P.H.; K. D. G. REID, † M.B., CH.B., D.P.H. \\ C. F. SPEIRS, $\ddagger$ M.B., CH.B., M.R.C.P.ED.
}

Brit. med. F., 1966, 1, 1400-1402

Bronchopulmonary geotrichosis is a rare disease caused by the fungus Geotrichum candidum. It has been described most commonly in South America, but has also been reported in North America, Scandinavia, France, and Great Britain. In describing what is probably the third case in the British literature, we draw attention to the possible seriousness of the illness. The severe asthma in this case is a feature not previously reported. The problems of management are described.

\section{Case Report}

In January 1963 a 46-year-old non-smoking previously healthy Edinburgh housewife who had never been abroad developed a severe expiratory wheeze and a cough initially productive of white sputum. During the next two months her general health deteriorated. Because the sputum had become purulent and copious her general practitioner prescribed tetracycline. As she did not improve, she was admitted to hospital on 14 April.

- Consultant Chest Physician, City Hospital, Edinburgh.

† Chest Physician, City Hospital, Edinburgh.

₹ Lately Senior House Officer, Department of Respiratory Diseases and Tuberculosis, City Hospital, Edinburgh.

\section{First Admission}

On admission, although apyrexial, she was exceedingly ill and wasted. An almost incessant cough produced copious green foulsmelling sputum, and a loud expiratory wheeze was audible to the unaided ear. Chest expansion was poor, and rhonchi and coarse crepitations were heard throughout both lung fields. There was probably early finger-clubbing. Haematological findings were: $\mathrm{Hb} 87 \%$; E.S.R. $30 \mathrm{~mm}$. in first hour (Westergren); W.B.C. $10,100 /$ c.mm. ( $10 \%$ eosinophils). Eosinophils were also present in the sputum. Sputum specimens were cultured aerobically and anaerobically on blood agar but no pathogens were isolated. Acidfast and alcohol-fast bacilli were not identified on direct smears. The Heaf test was grade III positive. A chest $x$-ray film revealed no definite abnormality other than a slight increase in left basal markings.

Her dyspnoea was not relieved by subcutaneous adrenaline, oral ephedrine, or intravenous aminophylline. Hypersalivation, which was a marked feature, was uninfluenced by oral atropine. As she continued to cough up $100 \mathrm{ml}$. of mucopurulent sputum daily, benzylpenicillin 1 mega unit six-hourly and streptomycin sulphate 0.5 g. 12-hourly, both intramuscularly, were given empirically from 19 to 27 April. As there was little change in her clinical state or in the character and volume of her sputum, chloramphenicol 500 
mg. six-hourly was given orally from 27 April to 3 May. There was no clinical benefit.

Because no bacteria had been isolated from her sputum, and in view of her asthma and eosinophilia in sputum and peripheral blood, the possibility of a fungal infection was considered. Skin tests for Aspergillus fumigatus and $A$. niger were negative. A pure growth of $G$. candidum was, however, isolated from the sputum on Sabouraud's medium. For two weeks the fungus continued to be isolated daily from the sputum and on several occasions was also isolated from the vagina and stool. On account of the possibility of an anaphylactic response resulting from nystatin inhalation as may occur in pulmonary aspergillosis with asthmatic features, this line of therapy was withheld. She was, however, given nystatin, 3 mega units daily orally for seven days, and the fungus was no longer isolated from the stool or vagina.

Previous to 21 May her average forced expiratory volume in one second (F.E.V.1) was $900 \mathrm{ml}$., her predicted normal being $1,840 \mathrm{ml}$. On this date, because of her severe dyspnoea and poor response to standard bronchodilators, it was necessary to begin treatment with prednisolone $5 \mathrm{mg}$. six-hourly orally. Within three days her breathing had improved greatly, her F.E.V.1 having risen to 1,400 $\mathrm{ml}$. Her daily sputum volume had decreased to $30 \mathrm{ml}$. and was mucoid. In order to determine whether the fungus was responsible for the patient's hypersensitivity state, a skin-testing solution was prepared commercially for her strain of geotrichum. On 6 June a scratch test by the standard method was strongly positive.

Any attempt to reduce her dose of prednisolone was associated with a recurrence of asthma and an increase in sputum volume. Finger-clubbing, suspected at the time of admission, was now a pronounced feature. Her arterial blood oxygen saturation before and after exercise was $92.5 \%$. Commercially prepared solutions of increasing concentration of the patient's fungus were given by subcutaneous injection in an attempted desensitizing process starting on 4 July, when her Geotrichum skin test was still strongly positive. Attempts to reduce the dose of prednisolone during the first two weeks of desensitization resulted in a recurrence of symptoms: thereafter it became possible to reduce the dose by $1 \mathrm{mg}$. daily. It was now apparent that the severe finger-clubbing was decreasing. At the end of the desensitizing course on 26 September she was symptomfree, her respiratory function tests were normal, geotrichum skin-sensitivity test was negative, and she did not require prednisolone. Bronchography on 2 October was normal. Daily sputum examination had not revealed the presence of $G$. candidum for a period of two months. She was discharged home on 19 October.

\section{Second Admission}

She was readmitted as an emergency case on 12 January 1964, having during the preceding three months developed symptoms similar to those on the previous occasion, treatment with standard bronchodilators by her general practitioner having proved unsuccessful. G. candidum was cultured daily from her sputum but the geotrichum skin test was negative. On admission her clinical condition necessitated treatment with prednisolone $30 \mathrm{mg}$. daily. Within 48 hours there was a dramatic improvement in her symptoms. It was not possible, however, to reduce her dose of prednisolone below $10 \mathrm{mg}$. daily without a recurrence of asthma. At bronchoscopy on 31 January the trachea and both stem bronchi were lined by a white fur, easily brushed off with a swab, from which a pure growth of $G$. candidum was obtained. Despite a negative geotrichum skin test, it was obvious that the desensitization procedure had not been fully effective in controlling her disease. The fungus was submitted to laboratory sensitivity tests covering a wide variety of antimicrobial agents and was found to be sensitive only to sulphonamides, colistin, nystatin, and framycetin, by the standard disc method. The organism was highly resistant to amphotericin B. It was decided to treat the patient with colistin methane sulphonate (Colomycin), and she was given 1 megaunit eight-hourly intramuscularly from 1 to 20 February. G. candidum was not cultured from the sputum after 11 February. At bronchoscopy on 24 February only a few white patches were seen and no growth was obtained from swabs on culture. On 5 February the patient's serum agglutinated $G$. candidum in a dilution of 1 in 30 . Information had emerged that a short time before the initial onset of symptoms in January 1963 the patient had been planting bulbs in bowls. G. candidum was cultured from the bulb-potting fibre, which the patient had kept at home for two years, whereas no fungus was isolated from many samples of fibre from other sources. On 19 March she was discharged home, symptom-free, on prednisolone 10 mg. daily.

During the next year her general health was satisfactory, though on one or two occasions she had mild asthmatic attacks associated with attempts to withdraw prednisolone. Occasionally a growth of G. candidum was obtained from laryngeal swabs.

\section{Third Admission}

She was readmitted to hospital on 4 March 1965 because of a moderate exacerbation of symptoms. Her skin. test for $G$. candidum was again positive, and a heavy growth of the fungus was cultured from her sputum. She was treated with colistin methane sulphonate 1 mega unit eight-hourly intramuscularly and also twice-daily inhalations of 0.5 mega unit by means of a Bennett respirator for two weeks. At the time of discharge on 3 April she was symptom-free, her respiratory function tests were normal, bronchoscopy was normal, and no fungus was isolated from laryngeal swabs. She was not receiving prednisolone at this time.

One month later, however, she had a recurrence of symptoms, and a scanty growth of the fungus was obtained from her sputum. Her symptoms were rapidly brought under control when she was given outpatient treatment with intramuscular colistin methane sulphonate for 12 days. Oral prednisolone was given concurrently, and its administration has been continued in a dose of $7.5 \mathrm{mg}$. daily.

Since then she has remained in excellent health with no clinical evidence of bronchospasm and is sputum-free. She is capable of moderate physical exertion, including riding a bicycle. Her average F.E.V.1 has remained at $1,650 \mathrm{ml}$., thus approaching the expected normal for a woman of her size and age. There have been occational scanty growths of $G$. candidum from laryngeal swabs and recently a few colonies resistant to colistin have been observed. The fungus is, however, fully sensitive to Pimaricin (natamycin), a drug against which the organism had not previously been tested. On 26 October the patient's serum agglutinated $G$. candidum in a dilution of 1 in 960 .

\section{Discussion}

The genus Geotrichum was defined by Link in 1809 to include fungi with truncate spores. He described only one species, G. candidum. The original description was modified by later workers, although the names of the genus and species were retained. Subsequently much confusion developed regarding the identifying features of the fungus, but the situation was made less obscure by Carmichael (1957), who described the habitat and cultural characteristics of 52 strains of $G$. candidum and also listed 55 synonyms found in older literature.

Although the fungus is usually regarded as saprophytic in earth and decaying organic matter and may be a commensal in man, it has been reported as causing a wide variety of pathological conditions such as tonsillitis, conjunctivitis, cutaneous lesions, ulcerative rectal lesions, septicaemia, and, most commonly, bronchopulmonary disease (Minton et al., 1954).

In reporting the first case of bronchopulmonary geotrichosis in British literature, Bell et al. (1962) quote Magalhaes, who stated that $G$. candidum was responsible for $1-1.5 \%$ of lung infections in Brazil. The condition is, however, rare in the Northern Hemisphere. There are no pathognomonic symptoms or signs of bronchopulmonary geotrichosis and it may simulate chronic bronchitis, pulmonary tuberculosis, or a lung abscess. Sputum, if present, may be earthy-smelling and of mucoid type with greyish flecks (Smith, 1934), and this is the description usually given in textbooks. Minton et al. (1954), however, describe one case with rusty sputum and another with frank haemoptyses, and the case reported by Bell et al. (1962) had a purulent sputum. Chest $x$-ray films are not diagnostic and simulate other diseases-for example, tuberculosis, bacterial pneumonia, other pulmonary mycoses, carcinoma, and chronic bronchitis (Webster, 1957). 
Kaliski et al. (1952) state that the organism should not be regarded as pathogenic unless found in large numbers in repeated fresh specimens of sputum. In our case the fungus was isolated from the sputum on 73 occasions and was also grown from the white fur seen lining the trachea and bronchi. Similar bronchoscopic findings have been described by Minton et al. (1954). As no other organism was isolated from the sputum at any time, we consider that our patient's clinical features were due to the fungus. In support of this conclusion it should be noted that during the course of the illness there was a thirtyfold rise in the patient's serum of Geotrichumagglutinating antibodies.

Human bronchopulmonary mycotic infections are usually secondary to other lung diseases-for example, tuberculosis and bronchiectasis. Other debilitating conditions, such as diabetes mellitus or the reticuloses, may be predisposing factors. Drugs such-as glucocorticosteroids and broad-spectrum antibiotics may sometimes be implicated when given over a prolonged period. In our patient's previous history only one of the above factors existed-namely, the administration of tetracycline for six weeks before her admission to hospital followed by treatment with the combination of penicillin and streptomycin and later chloramphenicol. It is significant that $G$. candidum was isolated from bulb fibre in the patient's home, whereas several other specimens of bulb fibre yielded no growth of the fungus.

Other pulmonary mycotic infections-for example, aspergillosis-may be associated with hypersensitivity phenomena. Kunstadter et al. (1946) mention two cases of chronic bronchitis with mild bronchospasm from whose sputum G. candidum was isolated, but they give no details of the cases. Kunstadter et al. (1950) describe cutaneous hypersensitivity to geotrichum extract in patients without asthma. Bell et al. (1962) describe the case of a 57-year-old man with bronchopulmonary geotrichosis who had several mild asthmatic attacks relieved by aminophylline injections. Our patient had no previous history of bronchitis or asthma and yet she developed severe persistent asthma which was not significantly relieved by ephedrine, adrenaline, and aminophylline in full dosage. She also had a strongly positive geotrichum skin-sensitivity test. High doses of prednisolone brought about subjective and objective improvement in respiratory function, and it would appear that a course of desensitizing injections of geotrichum extract was temporarily beneficial and permitted the reduction of the dose of prednisolone. It has not, however, been possible to eliminate her hypersensitivity entirely. Failure to eradicate the fungus is probably the important factor.

The treatment of pulmonary mycotic infections is difficult. Numerous antifungal agents are active in vitro, but few are effective in vivo. Most antifungal agents are effective only when applied locally to superficial lesions. Most are extremely toxic if given systemically, and, though they may be less toxic if given orally, they are also less effective (Edwards and $\mathrm{La}$ Touche, 1964). Many forms of therapy have been used for bronchopulmonary geotrichosis. Iodides have been used either as sodium iodide intravenously or saturated solutions of potassium iodide orally with limited success (Minton et al., 1954). Radiotherapy has been attempted by Kunstadter et al. (1950). Bendove and Ashe (1952) used neomycin intravenously to treat a patient who had developed geotrichum septicaemia, but clinical improvement was marred by the development of total deafness. Bell et al. (1962) treated a patient with potassium iodide orally and then with oral nystatin and nystatin inhalations for 12 weeks without eradication of the fungus. Brodie et al. (1962) isolated five strains of $G$. candidum which were sensitive to colistin and treated one patient with colistin methane sulphonate intramuscularly for 10 days with apparent success. They, however, later gave nystatin inhalations. Our patient's fungus has not been eradicated by colistin methane sulphonate intramuscularly nor by inhalations of the drug by a method similar to that used by Mensi (1958) in the treatment of other infections. None the less, when she had severe symptoms in association with a heavy growth of $G$. candidum from the sputum, treatment with the antibiotic was associated with disappearance of the fungus from the sputum and also improvement of bronchoscopic appearances. During two years the patient received 100 mega units of colistin methane sulphonate intramuscularly and 14 mega units by inhalation, but no side-effects were noted. Hutinel (1958) reported perfect tolerance when giving 90 mega units of this drug intramuscularly within one month in treating a patient with Escherichia coli endocarditis. As the patient's fungus later showed some resistance to colistin, we would probably use Pimaricin in the event of relapse.

\section{Summary}

The case is reported of a previously healthy 46-year-old woman who developed severe bronchitis and asthma due to the fungus Geotrichum candidum. The probable mode of infection is described. Previously described features of this rare disease, which are discussed, have not included severe asthma. The patient required prednisolone. An attempt was made to desensitize her with a course of solutions of increasing concentration of $G$. candidum extract, but the benefit was limited. The fungus was sensitive to colistin, but, though clinical improvement was achieved with the use of the drug, complete eradication of the fungus was not obtained. Colistin methane sulphonate was given intramuscularly alone and also in combination with inhalations. Treatment relieved severe manifestations, and at the time of writing the patient was symptom-free on a small daily dose of prednisolone.

We wish to express our thanks to Dr. Margaret Calder, who carried out bacteriological and mycological studies, and to Dr. W. Blyth for further mycological advice ; to Dr. Graham Crompton for bronchography; and to Mr. Philip Walbaum for the bronchoscopies. We are indebted to Dr. A. J. Jouhar, of Duncan, Flockhart \& Co. Ltd. (Edinburgh), for preparing the geotrichum skin-testing and desensitizing solutions.

\section{REFERENCES}

Bell, D., Brodie, J., and Henderson, A. (1962). Brit. F. Dis. Chest, 56, 26.

Bendove, R. A., and Ashe, B. I. (1952). Arch. intern. Med., 89, 107.

Brodie, J., Chambers, W., and Henderson, A. (1962). Lancet, 2, 300

Carmichael, J. W. (1957). Mycologia, 49, 820.

Edwards, G., and La Touche, C. J. P. (1964). Lancet, 1, 1349.

Hutinel, B. (1958). Thèse Paris, Exp. Scientifique, Ed. Paris.

Kaliski, S. R., Beene, M. L., and Mattman, L. H.'(1952). F. Amer. med. Ass., 148, 1207.

Kunstadter, R. H., Milzer, A., and Whitcomb, F. C. (1950). Amer. 7. Dis. Child., $79,82$.

Dendergrass, R. C., and Schubert, J. H. (1946). Amer. F. med. Sci., 211, 583.

Mensi, $\dot{\mathrm{E}}$. (1958). Minerva med. 49, 97.

Mensi, E. (1958). Minerva med Shanbrom, E. (1954). Ann. intern. Med., 40, 340 .

Smith, D. T. (1934). F. thorac. Surg., 3, 241.

Webster, B. H. (1957). Amer. Rev. Tuberc., 76, 286. 